\title{
Investigating the specificity and stoichiometry of RNA binding by the nucleocapsid protein of Bunyamwera virus
}

\author{
BJORN-PATRICK MOHL and JOHN N. BARR \\ Institute of Molecular and Cellular Biology, University of Leeds, Leeds LS29JT, West Yorkshire, United Kingdom
}

\begin{abstract}
Bunyamwera virus (BUNV) is the prototypic member of both the Orthobunyavirus genus and the Bunyaviridae family of negative stranded RNA viruses. In common with all negative stranded RNA viruses, the BUNV genomic and anti-genomic strands are not naked RNAs, but instead are encapsidated along their entire lengths with the virus-encoded nucleocapsid (N) protein to form a ribonucleoprotein (RNP) complex. This association is critical for the negative strand RNA virus life cycle because only RNPs are active for productive RNA synthesis and RNA packaging. We are interested in understanding the molecular details of how $\mathrm{N}$ and RNA components associate within the bunyavirus RNP, and what governs the apparently selective encapsidation of viral replication products. Toward this goal, we recently devised a protocol that allowed generation of native BUNV N protein that maintained solubility under physiological conditions and allowed formation of crystals that yielded high-resolution $\mathrm{x}$-ray diffraction data. Here we extend this work to show that this soluble $\mathrm{N}$ protein is able to oligomerize and bind RNA to form a highly uniform RNP complex, which exhibits characteristics in common with the viral RNP. By extracting and sequencing RNAs bound to these model RNPs, we determined the stoichiometry of N-RNA association to be $\sim 12$ nucleotides per $\mathrm{N}$ monomer. In addition, we defined the minimal sequence requirement for BUNV RNA replication. By comparing this minimal sequence to those bound to our model RNP, we conclude that $\mathrm{N}$ protein does not obligatorily require a sequence or structure for RNA encapsidation.
\end{abstract}

Keywords: Bunyamwera; RNA; encapsidation; replication; virus

\section{INTRODUCTION}

The Bunyaviridae family of negative-stranded RNA viruses comprises five genera, namely Orthobunyavirus, Hantavirus, Phlebovirus, Nairovirus, and Tospovirus. Bunyamwera virus (BUNV) is the prototypic member of both the Orthobunyavirus genus and the Bunyaviridae family as a whole, and serves as an excellent model to study the molecular and cellular biology of the serious human pathogens that reside within this group.

As with all bunyaviruses, the BUNV genome comprises three strands of RNA named small (S), medium (M), and large (L). These three BUNV segments act as templates for two distinctly different RNA synthesis activities, namely RNA replication and mRNA transcription. During replication, the negative stranded genome is copied to yield a perfectly complementary positive-stranded anti-genome,

Reprint requests to: John N. Barr, Institute of Molecular and Cellular Biology, University of Leeds, Leeds LS29JT, West Yorkshire, United Kingdom; e-mail: j.n.barr@leeds.ac.uk; fax: +44 (0)113 3433167.

Article published online ahead of print. Article and publication date are at http://www.rnajournal.org/cgi/doi/10.1261/rna.1367209. which acts as an intermediate for the generation of further genomic strands. During transcription, each genomic strand is copied to yield a single mRNA that possesses a capped $5^{\prime}$ extension, and is truncated at its $3^{\prime}$ end relative to the negative stranded template. The $\mathrm{S}$ mRNA encodes the $\mathrm{N}$ and NSs proteins from overlapping reading frames (Fuller and Bishop 1982; Fuller et al. 1983; Elliott 1989b); the M mRNA encodes a polyprotein that is cleaved to yield the Gn and Gc envelope proteins, and nonstructural NSm (Gentsch and Bishop 1979; Fuller and Bishop 1982; Elliott 1985); and the L mRNA encodes the L protein (Elliott 1989a) that is the RNA-dependent RNA polymerase (RdRp), responsible for all viral RNA synthesis activities.

In common with all negative stranded RNA viruses, the BUNV genomic and anti-genomic templates are not naked RNAs, but instead are encapsidated along their entire lengths with the virus-encoded $\mathrm{N}$ protein to form a ribonucleoprotein (RNP) complex. Within the RNP, the $\mathrm{N}$ protein sequesters the RNA such that the phosphate backbone is partially protected from the solvent, whereas the bases are exposed (Baudin et al. 1994; Iseni et al. 2000, 2002). The molecular basis for this mode of binding is now 
supported by recent atomic resolution structural data for the $\mathrm{N}$ proteins of vesicular stomatitis virus (VSV) (Green et al. 2006), rabies virus (Albertini et al. 2006), and influenza virus (Ye et al. 2006). These data show that the phosphate backbone of the RNA binds within a specialized cavity that is lined with amino acids including positively charged side chains, with aromatic groups also playing a role in RNA binding by stacking with the RNA bases. For this group of viruses, the process of encapsidation results in the intimate association of the RNA strand with a continuous chain of $\mathrm{N}$ protein monomers: Each $\mathrm{N}$ protein monomer is in contact with the same number of RNA nucleotides (Albertini et al. 2006; Green et al. 2006), and this association is repeated along the length of the replication product. This association is critical for the negative strand RNA virus life cycle because it is only in the form of the RNP that the genomic and anti-genomic strands are active for productive RNA synthesis. The intimacy of the N/RNA association is demonstrated by RNPs derived from members of the subfamily Paramyxovirinae, in which the relative position of the 6 nucleotides (nt) that binds each $\mathrm{N}$ monomer (Egelman et al. 1989) directly affects recognition of cis-acting signals on the template by the RdRp (Iseni et al. 2002). In addition, the N/RNA association is critical to the life cycle because only encapsidated RNAs are packaged into virus particles.

It is generally accepted that encapsidation of negative strand RNA virus replication products begins at the $5^{\prime}$ terminus, and proceeds concurrently with RNA chain elongation until the entire RNA strand is enwrapped, and there is some direct evidence of this (Gubbay et al. 2001). Consistent with the functional requirements of this model, the BUNV $\mathrm{N}$ protein is known to both tightly associate with single stranded RNA (Osborne and Elliott 2000) and also to associate homotypically and form higher-order oligomers (Leonard et al. 2005). These abilities in combination have been suggested to allow successive $\mathrm{N}$ protein units to bind to the growing RNA strand through combined affinity toward both RNA and $\mathrm{N}$ protein within the partially assembled RNP.

However, a long-standing question in RNA virology is what governs the selection of the RNA molecule to be encapsidated, such that viral replication products are selectively encapsidated as opposed to RNAs of a cellular origin. Several possible explanations have been proposed, including the presence of encapsidation signals on nascent replication products, functional coupling between replication and encapsidation (Nugent et al. 1999) or colocalization of replicating RNAs with $\mathrm{N}$ protein production (Venter et al. 2005). Toward answering this question for BUNV, competitive binding assays have shown that the BUNV $\mathrm{N}$ protein exhibits preferential binding for RNAs that contain sequences represented within the nontranslated region (NTR) of the genomic $5^{\prime}$ end (Osborne and Elliott 2000). Binding was shown to be most specific for
RNAs that comprised nucleotides $1-33$ of the genomic 5' NTR, and it was proposed that secondary structures within these sequences might provide the basis for binding the BUNV replication products.

Signals that promote encapsidation of replication products have also been reported for other bunyaviruses, most notably for those classified within the Hantavirus genus. In separate studies, the Hantaan virus $\mathrm{N}$ protein was shown to exhibit binding preference for both $5^{\prime}$ genomic sequences (Severson et al. 2001) and also for panhandle structures that arise through base pairing between the complementary nucleotides within $3^{\prime}$ and $5^{\prime}$ terminal regions (Severson et al. 2001; Mir and Panganiban 2004, 2005), and it was proposed that these structures nucleated initial binding of a $\mathrm{N}$ protein trimer, after which RNP elongation was achieved by addition of further preformed trimers in a sequence independent manner.

We are interested in understanding the molecular details of how $\mathrm{N}$ protein and RNA components associate within the bunyavirus RNP. In this report, we focused on investigating the initial binding stage of encapsidation, where $\mathrm{N}$ protein first binds free RNA. Toward this goal, we recently devised a protocol that allows expression and purification of native BUNV $\mathrm{N}$ protein that maintained solubility under physiological conditions (Rodgers et al. 2006). This material was of high purity and stability, and allowed formation of crystals that yielded high-resolution $\mathrm{x}$-ray diffraction data.

Here we extend this work to show that this soluble $\mathrm{N}$ protein is able to oligomerize and reversibly bind RNA to form a highly uniform RNP complex, which exhibits characteristics in common with the viral RNP. By extracting and sequencing the RNAs bound to these model RNPs we have been able to determine the stoichiometry of $\mathrm{N}$ RNA association. In addition, we defined the minimal sequence requirement for BUNV RNA replication, and by comparing this minimal sequence to sequences bound to our model RNP, we have been able to examine whether the $\mathrm{N}$ protein exhibits any obligate requirements for RNA encapsidation.

\section{RESULTS AND DISCUSSION}

\section{Using gel filtration to determine the oligomeric state of the BUNV $\mathbf{N}$ protein in solution}

We previously determined growth and harvesting conditions that allowed large-scale production of native BUNV N protein that was soluble under native conditions (Rodgers et al. 2006). This material was used to generate $\mathrm{N}$ protein crystals that successfully diffracted $\mathrm{x}$ rays to a resolution in excess of $3 \AA$, and these data are currently being used for solution of the BUNV N protein three-dimensional atomic structure. We next wanted to use this material for further analysis of the behavior of the BUNV N protein in solution, 
in particular to investigate its oligomeric state, which may provide information of how the $\mathrm{N}$ protein assembles into multimeric RNP structures. To achieve this aim, we first used gel filtration to determine the molecular weight of the soluble species.

BUNV N protein was expressed and purified as described previously (Rodgers et al. 2006), and subjected to gel filtration (Fig. 1A). The majority of applied protein eluted as two peaks; a minor peak of early-eluting material that
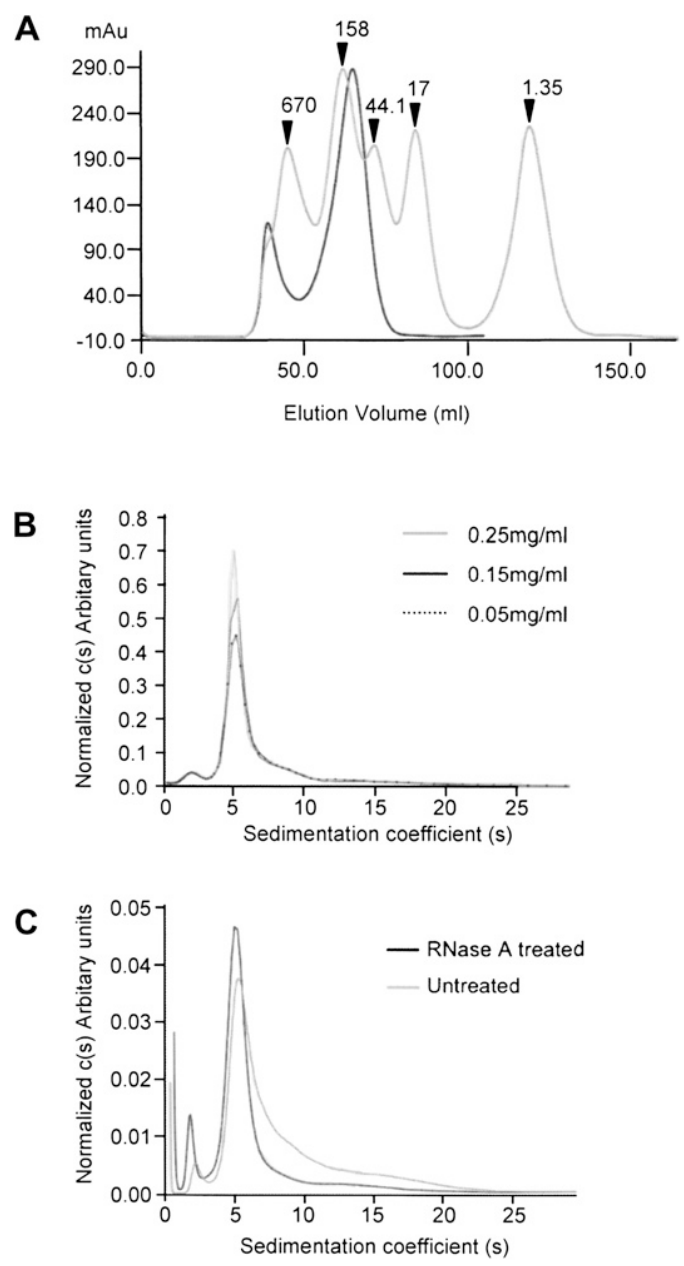

FIGURE 1. Analysis of the BUNV N/RNA complex using gel filtration and analytical ultracentrifugation. (A) Bacterially expressed His-tagged BUNV N protein was purified by nickel affinity chromatography, after which the His-tag was removed by thrombin cleavage and applied to a Superdex S300 size exclusion column at a flow rate of $1 \mathrm{~mL} / \mathrm{min}$. The elution profile of the $\mathrm{N}$ protein is shown in black, and the profile of known molecular weight standards applied to the same column is shown in gray. $(B)$ Three different concentrations of the BUNV $\mathrm{N}$ protein were analyzed using analytical sedimentation centrifugation at 40,000 rpm for $14 \mathrm{~h}$. Sedimentation was measured using absorbance optics at $260 \mathrm{~nm}$, and the resulting data analyzed using SEDFIT as described in Materials and Methods. (C) N/RNA complexes at a concentration of $0.25 \mathrm{mg} / \mathrm{mL}$ were subjected to complete RNase A digestion, and analyzed by analytical sedimentation centrifugation alongside undigested samples. Sedimentation was measured and analyzed as described above. was likely insoluble protein aggregates, and a major peak of late-eluting material. By comparing the elution of the BUNV N protein with molecular weight standards, we calculated the predominant species to have a molecular weight of $109 \mathrm{kDa}$. When considering the molecular weight of $\mathrm{N}$ protein monomers as $26.945 \mathrm{kDa}$ (Rodgers et al. 2006), this closely corresponded to a tetramer complex $(107.8 \mathrm{kDa})$.

\section{Confirmation of the oligomeric state of the BUNV N protein using analytical ultracentrifugation}

To provide independent confirmation of the molecular weight of the $\mathrm{N}$ protein oligomer, we next subjected the $\mathrm{N}$ protein to both analytical sedimentation and analytical equilibrium ultracentrifugation. In addition, these techniques would allow us to investigate whether the $\mathrm{N}$ protein exhibited concentration-dependent changes in oligomerization state. The $\mathrm{N}$ protein used in both these experiments was expressed and purified as described previously (Rodgers et al. 2006).

Sedimentation centrifugation was performed at 50,000 rpm on three separate $\mathrm{N}$ protein samples with nominal concentrations of $0.25,0.15$, and $0.05 \mathrm{mg} / \mathrm{mL}$, as described in Materials and Methods. Movement of the $\mathrm{N}$ protein was measured using both absorbance and interference optics, and overlays of scans obtained using both methods indicated that the $\mathrm{N}$ protein was complexed with RNA, presumably originating from the host bacterial cells used to express the protein.

From the plateau height measurement, and the extinction coefficient of BUNV N protein and RNA, the relative proportion of protein to RNA was calculated to be $14.4 \mathrm{~g} / \mathrm{g}$, which allowed us to calculate a partial specific volume for the N-RNA complex using SEDNTERP (http://www.rasmb. bbri.org/). The raw sedimentation data were then used to determine sedimentation coefficient distributions for the N-RNA complex using SEDFIT (Schuck 2000), and plots of the distributions are shown in Figure 1B. Both absorbance and interference data sets resulted in similar distributions: A major component of $\sim 5 \mathrm{~S}$, and a minor shoulder of material between 7 and 10S, which likely represents higherorder assemblies of the $\mathrm{N}$ protein. At the nominal N-RNA concentrations of $0.25,0.15$ and $0.05 \mathrm{mg} / \mathrm{mL}$, the major $5 \mathrm{~S}$ peaks corresponded to a molecular weight of $124 \mathrm{kDa}$ ( \pm 12$), 122 \mathrm{kDa}( \pm 3.2)$, and $124 \mathrm{kDa}( \pm 11)$, respectively. Based on the relative proportion of protein mass to RNA mass determined as described above (14.4/1), the contribution of the RNA component was calculated to be 8.5 $\mathrm{kDa}$. By subtracting this amount, the protein component alone was calculated to be $\sim 114.5 \mathrm{kDa}$, and the nearest integer oligomer to this amount is a tetramer $(107.8 \mathrm{kDa})$. Interestingly, the relative abundances of the various peaks of sedimenting material were superimposable at each of the three concentrations examined, indicating that within this 
concentration range, there was no significant dynamic interchange between the various $\mathrm{N}$ protein oligomers.

We also performed equilibrium centrifugation at speeds of 9000 and 14,000 rpm on three separate N-RNA samples with nominal concentrations of $0.25,0.10$, and $0.05 \mathrm{mg} /$ $\mathrm{mL}$. The raw equilibrium data for both speeds were analyzed and combined using SEDANAL (Stafford and Sherwood 2004) according to a single component model, which calculated the molecular weight to be $138 \mathrm{kDa}$ $( \pm 16)$. As described above, by subtracting the mass of RNA component based on the relative proportion of protein to RNA (14.4/1) the mass of the protein component was calculated as $128 \mathrm{kDa}$. This was in good agreement with the estimated molecular weight as determined by sedimentation centrifugation, described above.

\section{The oligomeric state of the BUNV N protein does not depend on the presence of RNA}

To determine whether the oligomeric state of the BUNV N protein relied upon the presence of RNA, we removed the RNA component using RNase A digestion, and analyzed the resulting protein component using analytical sedimentation centrifugation. A comparison between the sedimentation profile of the $\mathrm{N}$ protein complex before and after RNase A digestion is shown in Figure 1C, which shows that the $\mathrm{N}$ protein in both samples existed as a major species having an $S$ value of $\sim 5 S$. Interestingly, the major peak in the RNase A-treated sample was of a slightly reduced $\mathrm{S}$ value, which likely resulted from loss of the RNA component, correspondingly reducing its mass. In addition, RNase A treatment reduced the abundance of material with $S$ values between 7 and $10 \mathrm{~S}$ described above and shown in Figure 1B. Taken together, these results suggest that while RNA was not needed for maintaining the $\mathrm{N}$ protein as a tetramer, it may facilitate further association of $\mathrm{N}$ protein into structures of higher molecular weights. While this analysis could not definitively determine whether these structures were higherorder oligomers or whether they were multiple tetramers bound to the same strand of RNA, it suggests that they were the latter due to the consolidation of material into a single peak following RNase digestion. In conclusion, the native BUNV $\mathrm{N}$ protein forms a tetramer in solution, which is the dominant species, with monomeric or defined higher-order oligomers being undetectable. The existence of BUNV N protein tetramers has previously been reported, identified by cross-linking $\mathrm{N}$ protein transiently expressed within mammalian cells (Leonard et al. 2005). However, multiple alternative oligomers were also detected including dimers, trimers, and possibly hexamers, which were not readily detected in our system. The reason why these two systems should generate different oligomeric states of the $\mathrm{N}$ protein is currently unknown.

\section{Analysis of the stoichiometry of RNA bound to the $\mathrm{N}$ protein}

To gain a better understanding of the structure of the BUNV RNP complex, we wanted to determine the stoichiometry of binding between $\mathrm{N}$ protein and RNA nucleotides. This is a fundamental structural feature of the BUNV RNP, and is currently undetermined for any member of the Bunyaviridae family. To achieve this aim, we needed to determine the nucleotide length of RNAs extracted from purified $\mathrm{N}$ protein tetramers. This was accomplished by harvesting RNA from $\mathrm{N}$ protein tetramers and then subjecting the RNA to electrophoresis on both polyacrylamide (Fig. 2A) and agarose gels (Fig. 2B) alongside appropriate RNA size markers. Using both gel systems, the majority of the harvested RNAs migrated as a prominent and discrete band that migrated alongside the $50 \mathrm{nt}$ size marker. With reduced RNA loading (Fig. 2A, lane 3), a more accurate assessment of RNA mobility could be made, which indicated the band was migrating slightly faster than the $50 \mathrm{nt}$ marker, at $\sim 48 \mathrm{nt}$. This length of RNA would correspond to a stoichiometry of $12 \mathrm{nt}$ per $\mathrm{N}$ protein monomer. A low abundance of larger RNAs were also detected, with regions of greater intensity evident at $\sim 100$ and $150 \mathrm{nt}$. These RNAs were likely components of the high molecular weight N/RNA complexes that we detected using both gel filtration and AUC analysis.

Interestingly, the N/RNA stoichiometry of BUNV is different from all other negative strand RNA viruses for which this information is known. Each $\mathrm{N}$ protein of the paramyxovirus Sendai virus binds 6 nt (Egelman et al. 1989), the $\mathrm{N}$ proteins of rhabdoviruses rabies virus and VSV bind 9 nt (Albertini et al. 2006; Green et al. 2006), and
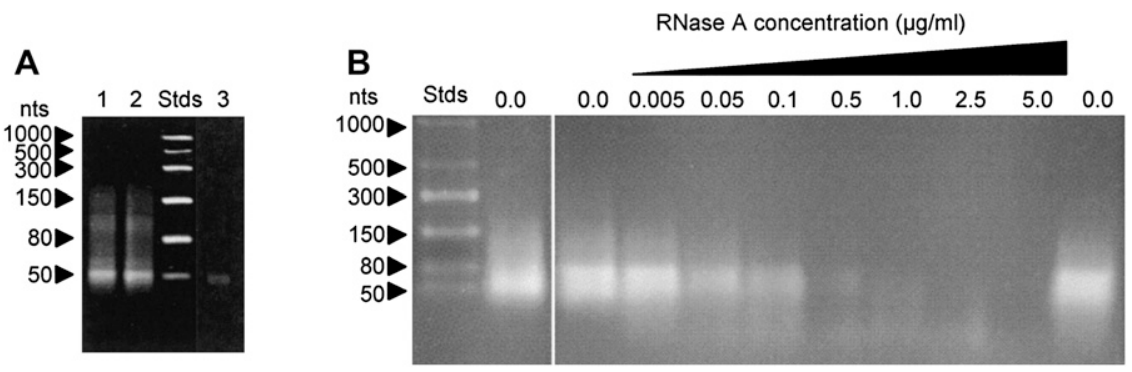

FIGURE 2. Analysis of RNA species bound to BUNV N protein tetramers. (A) RNA was harvested from purified tetramers and subjected to electrophoresis on a $10 \%$ denaturing polyacrylamide gel, alongside single strand RNA size markers. Lanes 1 and 2 are duplicate lanes, and lane 3 shows reduced loading. (B) N/RNA tetramers were incubated with increasing quantities of RNase A, after which the RNA was extracted and electrophoresed on a $1.5 \%$ nondenaturing agarose gel alongside single strand RNA size markers and undigested RNA. 
the orthomyxovirus influenza virus $\mathrm{N}$ protein binds $24 \mathrm{nt}$ (Ortega et al. 2000). The structural basis for the defined stoichiometry of RNA binding of rhabdovirus and orthomyxovirus $\mathrm{N}$ proteins is now well understood due to the recent determination of their respective crystal structures, which have shown it is due, in a major part, to the size of the RNA binding cavity and positioning of charged and aromatic side chains that lie within (Albertini et al. 2006; Green et al. 2006). Therefore, our results suggest that the BUNV N protein may possess an RNA binding cavity that exhibits significant differences to these other $\mathrm{N}$ proteins.

To compare the biochemical properties of our soluble NRNA preparation with both authentic bunyavirus RNPs harvested from infected cells (Hacker et al. 1989, 1990), and similar BUNV RNPs formed within bacterial cells from transient N protein expression (Osborne and Elliott 2000), we examined the resistance of the bound $48 \mathrm{nt}$ fragment to RNase A digestion. We showed that the bound RNA was completely digested with an RNase A concentration of $5 \mu \mathrm{g} / \mathrm{mL}$ (Fig. 2B), which corresponded with previous determinations of RNase A resistance of these other RNP complexes, and indicated that all have similar properties. At lower concentrations of RNase A, the 48 nt RNA band was diminished in intensity, but RNAs of shorter lengths were not detected. This suggested that the 48 nt RNA strand was equally susceptible to RNase A digestion along its entire length.

\section{Analysis of RNA bound to the tetramers}

In the previous section, we showed that the BUNV $\mathrm{N}$ protein tetramer bound RNA strands of $\sim 48 \mathrm{nt}$ in length. We next wanted to investigate whether these RNAs possessed features that facilitated their binding to the $\mathrm{N}$ protein. As the pool of RNAs within the bacterial cell comprises a multitude of RNA species, we reasoned that the expressed $\mathrm{N}$ protein would be exposed to multiple RNAs as potential binding substrates. We hypothesized that if the bound RNAs had been selected by virtue of specific sequences or secondary structures that caused their binding over all other RNAs, these characteristics would be common to all bound RNAs.

To test our hypothesis, we wanted to isolate and sequence the bound RNAs to investigate whether they contained any common sequences or structures. Furthermore, we anticipated that if an essential encapsidation signal existed, it would likely be located proximal to the 5' end of each of the bound RNAs. This is because in the generally accepted unidirectional encapsidation model described above, $\mathrm{N}$ protein is proposed to first bind to an encapsidation signal and then enwrap RNA in the $5^{\prime}$ to $3^{\prime}$ direction. It is reasonable to assume that a similar process could also operate in the interior of the bacterial cell.

RNAs were extracted from the purified $\mathrm{N}$ protein tetramers, transcribed into cDNAs and subjected to sequence analysis. To reduce the possibility that any common features had arisen through chance, we sequenced a total of 59 RNAs, and these sequences are shown in Supplementary Table 1. As expected, all sequences were found to correspond to RNAs generated within the bacterial cells used for $\mathrm{N}$ protein expression, as determined by searching against known sequences within the Entrez nucleotide database. Consistent with our previous analysis of the bound RNA using gel electrophoresis, the most abundant group of RNAs was between 40 and $50 \mathrm{nt}$ long (25/59). Another group of RNAs having lengths approximately twice this (80-100 nt) was also evident, but were present at a reduced abundance (10/59), and these may have represented RNAs bound to higher-order oligomers. Interestingly, most of the remaining sequences were of less than $40 \mathrm{nt}$, and RNAs of this size were not abundantly detected during our analysis of the extracted RNA by gel electrophoresis. To account for this discrepancy, we propose that some sequence information may have been lost during the RT-PCR steps, most likely as a result of the anchored oligo $\mathrm{d}(\mathrm{T})$ primer annealing to A-rich sequences at the $3^{\prime}$ end of some RNAs. However, this possibility would not affect our search for common features within these RNAs, as the sequences we were most interested in were $5^{\prime}$ proximal, which were intact as evidenced by the presence of the entire adapter RNA within each of the cloned cDNA sequences.

To aid in our search for a common sequence motif involved in encapsidation, we reasoned that if an encapsidation signal existed, it must also reside within the $5^{\prime}$ ends of both BUNV genomic and anti-genomic RNAs. Therefore, when we aligned the 59 bound RNA sequences, we also included the BUNV 5' NTRs in the analysis. Furthermore, as our previous work showed that just $25 \mathrm{nt}$ at the $5^{\prime}$ end of the $\mathrm{S}$ segment is sufficient for replication and thus encapsidation of either BUNV genomic or anti-genomic strands (Barr et al. 2005), we included only these nucleotides in the alignment. Our analysis failed to detect any common motifs within these sequences, and thus suggested that the bound RNAs were unlikely to be encapsidated by virtue of a specific signal formed by a linear nucleotide sequence.

\section{Defining the minimal promoter for replication and encapsidation}

There is precedent within diverse viral systems for involvement of secondary structures in the association between genomes and capsid proteins during genome encapsidation or packaging. In some instances this association has been resolved to the molecular level, for example, in tobacco mosaic virus (Namba et al. 1989) and bacteriophage MS2 (Valegard et al. 1994). Therefore, we next wanted to examine whether encapsidation of the bound RNAs by the BUNV $\mathrm{N}$ protein required the obligatory presence of a defined secondary structure. 
As above, to aid in our search for these structures, we reasoned that if an encapsidation signal existed, it would reside within the $5^{\prime}$ ends of both BUNV genomic and antigenomic strands. Therefore, our strategy was to use M-fold analysis to identify possible structures within the BUNV 5' NTRs, and then search the bound RNAs for equivalent structures. To increase the accuracy of this analysis, we also wanted to eliminate nucleotides from the $\mathrm{M}$-fold analysis that were irrelevant to the encapsidation process. To achieve this aim we undertook a functional analysis of the BUNV replication promoter to precisely define the minimum sequence required for BUNV replication and hence, encapsidation.

We constructed plasmids that expressed model BUNV segments with progressive internal deletions within the NTRs of their genomic and anti-genomic strands, as shown in Figure 3, A and B. The parental construct for these deletions was the previously described model segment 25/25 (Barr et al. 2005), which contained the first 25 nt of both $3^{\prime}$ and 5' S segment NTRs, and which replicated with an ability approaching that of the wild type. A total of five model segments having deletions within their anti-genomic $3^{\prime}$ ends were constructed and were designated 25/23, 25/21, 25/18, and $25 / 17$, with the numbers corresponding to the lengths of the $5^{\prime}$ and $3^{\prime}$ anti-genomic NTRs, respectively. Using this

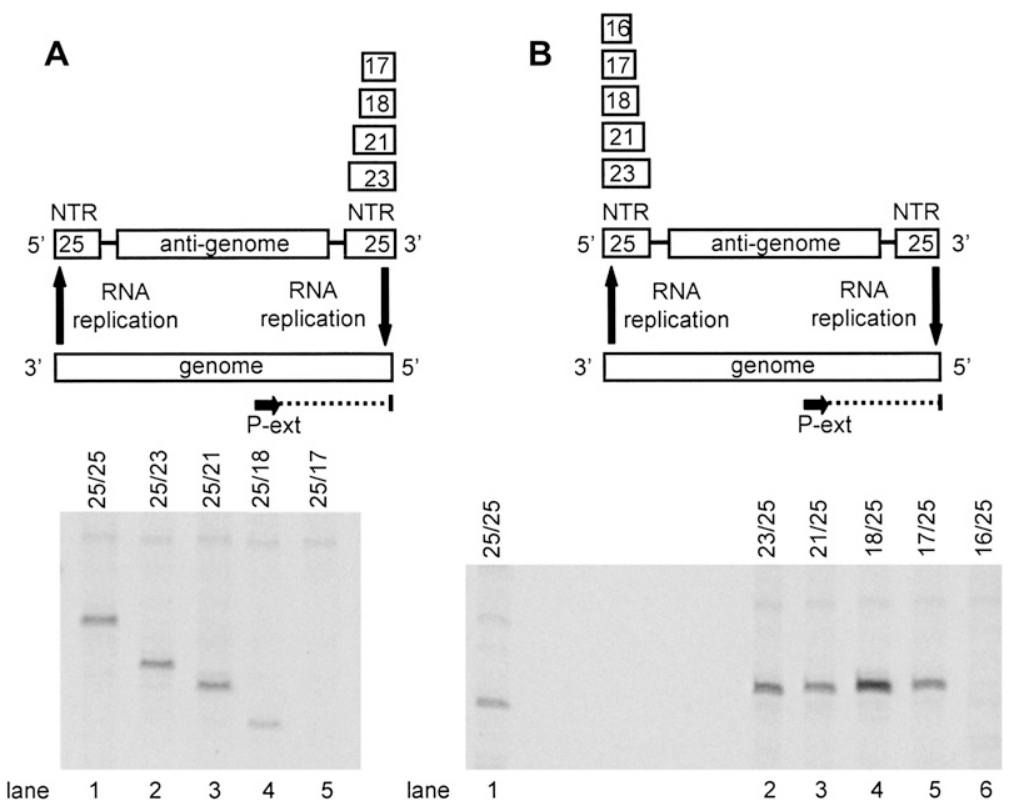

FIGURE 3. Schematic of model BUNV segments used to determine the minimal sequences required for RNA replication and primer extension analysis of the replication products they generate. $(A)$ Model BUNV segments were generated such that progressive internal nucleotide deletions were introduced into the $3^{\prime}$ NTR of the anti-genomic strand. The boxed numbers within the $3^{\prime}$ NTR denote remaining terminal nucleotides. After RNA replication within mammalian cells, these deletions were present within the corresponding $5^{\prime}$ NTR of the genomic strand. Abundance of genomic strands was determined by primer extension analysis using oligonucleotide P-ext, which binds within the body of all altered model segments. $(B)$ Progressive internal deletions were also engineered into the anti-genomic $5^{\prime}$ NTR, and its replication ability was determined as described above. same numbering scheme, a panel of six model segments having internal deletions within the $5^{\prime}$ anti-genomic NTR were also generated, and were named 23/25, 21/25, 18/25, $17 / 25$, and $16 / 25$. The replication ability of these segments was then determined by quantifying the amount of replication products they generated using primer extension analysis. This analysis showed that BUNV segments were active for replication with only $18 \mathrm{nt}$ within the anti-genomic $3^{\prime}$ NTR (Fig. 3A, lane 4), and only 17 nt within the genomic 5' NTR (Fig. 3B, lane 5). Primer extension analysis to quantify anti-genomic RNAs also was performed and resulted in the same outcome (data not shown).

\section{M-fold analysis of the replication promoters and bound RNAs}

In the previous section, we showed that $18 \mathrm{nt}$ within the antigenomic $3^{\prime}$ NTR and 17 nt within the anti-genomic 5' NTR were sufficient to signal replication. These results imply that if encapsidation signals exist, they must reside within the corresponding nucleotides within the $5^{\prime}$ end of the nascent replication products. To search for possible signals within the nomic and anti-genomic $5^{\prime}$ NTRs, these nucleotides were subjected to M-fold analysis, which predicted secondary structures having respective delta $\mathrm{G}$ values of $-1.6 \mathrm{kcal} / \mathrm{mol}$ and $-1.2 \mathrm{kcal} / \mathrm{mol}$, and which are shown in Figure 4, A and B, respectively. Interestingly, the structure predicted to fold in the genomic 5' NTR (Fig. 4A) was identical to the structure previously suggested to be required for encapsidation, based on the results of a competitive binding assay (Osborne and Elliott 2000).

We next wanted to see whether either of the two structures shown in Figure 4 was present within the bound RNAs. According to our hypothesis, if these signals were required for encapsidation, then they would be present within all the bound sequences (Supplementary Table 1). We first analyzed the 20 sequences that fell into the 40-50 nt size range. However, M-fold analysis failed to identify any structures formed by the genomic 5 ' end (Fig. 4A), and only seven possible instances of the structure formed by the antigenomic 5' end (Fig. 4B). Because neither structure was present in the majority of these RNAs, no further bound sequences were analyzed. Thus, our results suggest that the bound RNAs harvested from the $\mathrm{N}$ protein tetramers were not encapsidated by virtue of any obligatory signals, neither a linear sequence, nor a secondary structure. 


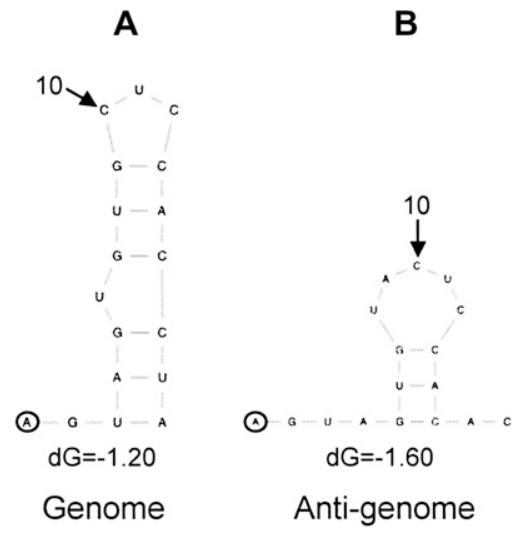

FIGURE 4. Schematics of the most energetically favorable secondary structures predicted by $\mathrm{M}$-fold to form in the $5^{\prime}$ end of the $(A)$ genomic and $(B)$ anti-genomic strands of the BUNV S segment. The terminal strand of each nucleotide is circled.

However, this conclusion does not exclude the possibility that BUNV replication products contain a signal that allows preferential binding over other RNAs. We are currently using the experimental system described here to investigate this possibility by incubating RNA-free $\mathrm{N}$ protein tetramers with a limited pool of defined RNAs, and then identifying and quantifying bound RNAs by sequence analysis.

Furthermore, if an encapsidation signal that drives preferential binding does exist, it will be interesting to understand how it is recognized by the RNA-binding groove within the BUNV $\mathrm{N}$ protein, particularly if the signal should comprise a secondary structure that includes a region of duplex RNA. In the case of VSV, the conformation of RNA within the RNA binding cleft was suggested to preclude the formation of Watson-Crick pairings, and so it is difficult to see how an encapsidation signal that includes secondary structure can be accommodated (Albertini et al. 2006; Green et al. 2006). Whether the same constraint is true of the BUNV $\mathrm{N}$ protein is currently unknown. However, we are hopeful that on-going structure solution of the BUNV N protein, along with bound RNA, will reveal molecular details of this, and other aspects of the N/RNA association, which are so critical to the viral life cycle.

\section{MATERIALS AND METHODS}

\section{Analysis of the purified BUNV N protein}

To determine the molecular weight of the native BUNV N protein in solution, the BUNV $\mathrm{N}$ protein generated in bacterial cells (Rodgers et al. 2006) was subjected to size exclusion chromatography, along with known molecular weight standards. Purified $\mathrm{N}$ protein in $150 \mathrm{mM} \mathrm{NaCl}$ and $20 \mathrm{mM}$ Tris- $\mathrm{Cl}$ (pH7.9) was injected onto a Superdex S300 column (GE-Healthcare Biosciences) and eluted protein detected using an AKTA explorer (GE-Healthcare Biosciences).
Molecular weight determination of the soluble $\mathrm{N}$ protein was also made using analytical sedimentation and analytical equilibrium centrifugation using an Optima XL-I ultracentrifuge (BeckmanCoulter). For sedimentation centrifugation, $0.42 \mathrm{~mL}$ of both test and reference samples were loaded into two-sector aluminum centerpiece cells, and centrifuged in an eight-place An-50 titanium analytical rotor at $40,000 \mathrm{rpm}$ at $21^{\circ} \mathrm{C}$. Three different concentrations of the $\mathrm{N}$ protein were analyzed $(0.25,0.15$, and $0.05 \mathrm{mg} /$ $\mathrm{mL}$, in $150 \mathrm{mM} \mathrm{NaCl}$ and $20 \mathrm{mM}$ Tris- $\mathrm{Cl} \mathrm{pH} \mathrm{7.9),} \mathrm{and} \mathrm{where}$ indicated, RNA was digested from the complex by incubation with $50 \mu \mathrm{g} / \mathrm{mL}$ of RNase A. Digestion was confirmed as complete by the demonstration that RNA could no longer be harvested from the complex using phenol and chloroform extraction followed by ethanol precipitation. Sedimentation of the N-RNA complex was measured using both absorbance $(260 \mathrm{~nm})$ and Rayleigh interference optics, and a total of 99 scans were performed over a run time of $14 \mathrm{~h}$. Buffer densities, viscosities, and partial specific volumes were calculated using the program SEDNTERP (http:// www.rasmb.bbri.org/), and the raw data were analyzed by wholeboundary profile analysis using the program SEDFIT (Schuck 2000).

For equilibrium centrifugation, $0.1 \mathrm{~mL}$ of test and reference samples were loaded into six-sector Epon centerpiece cells, and centrifuged in a four-place An-60 titanium rotor at either 9000 or $14,000 \mathrm{rpm}$ at $5^{\circ} \mathrm{C}$. Radial $260 \mathrm{~nm}$ absorbance scans (20 replicates, radial step size 0.001 $\mathrm{cm}$ ) were taken at $4 \mathrm{~h}$ intervals, and were judged to be at equilibrium by plotting radial offset vs. time using the program WinMatch. The raw equilibrium data for both speeds were analyzed and combined using SEDANAL (Stafford and Sherwood 2004).

\section{RNA extraction and analysis}

Total RNA was harvested from the N-RNA complex by sequential extractions using equal volumes of phenol and chloroform, followed by ethanol precipitation. To determine the size of the harvested RNA, samples were electrophoresed on both $1.5 \%$ nondenaturing agarose and $10 \%$ denaturing polyacrylamide gels, and compared to the mobility of low range RNA size markers (New England Biolabs) run in adjacent lanes. RNAs were stained with ethidium bromide, and visualized using long wavelength UV.

To clone the RNA and determine its nucleotide sequence, extracted RNA was modified by first tailing the $3^{\prime}$ ends using poly(A) polymerase (Applied Biosystems/Ambion) and then ligating an adapter RNA (5'-GCUGAUGGCGAUGAAUGAACAC UGCGUUUGCUGGCUUUGAUGAAA-3'; Applied Biosystems, Ambion) to the $5^{\prime}$ ends using T4 RNA ligase (Applied Biosystems, Ambion). The resulting RNAs were then used as templates for Moloney-murine leukemia virus reverse transcriptase-directed RT-PCR using an anchored oligo d(T) primer and inner primer (5'-CGCGGATCCGAACACTGCGTTTGCTGGCTTTGATG-3') that annealed within the ligated adapter. The amplified RT-PCR products were blunt-end ligated into plasmid pZero (Invitrogen), and inserts were sequenced using flanking oligonucleotide primer M13-reverse.

\section{Nucleotide sequence analysis}

cDNA sequences were viewed and edited using four-peaks software (A. Griekspoor and Tom Groothuis; mekentosj.com) and were aligned using Vector NTI software (Invitrogen). For identification of possible secondary structures, RNA sequences were analyzed using 
M-fold software (Zuker 2003) with parameters set as follows: The maximum interior loop size and asymmetry of an interior loop were $30 \mathrm{nt}$, and the maximum distance between paired bases was $18 \mathrm{nt}$ and $17 \mathrm{nt}$ for the genomic and anti-genomic $5^{\prime}$ NTR, respectively.

\section{Expression of truncated model segments}

All plasmids designed to express model BUNV segments having progressively truncated NTRs were derived from previously described plasmid pBUN-S(ren), which contained the entire $3^{\prime}$ and $5^{\prime}$ NTRs of the BUNV S segment surrounding the Renilla luciferase open reading frame (Barr et al. 2003). This plasmid was subsequently modified to generate plasmid p25/25, which expressed a model segment containing the first $25 \mathrm{nt}$ of $5^{\prime}$ and $3^{\prime}$ NTRs of the S segment anti-genomic strand (Barr et al. 2005). Further truncations were made such that nucleotides were progressively removed from the terminal-distal side of the NTRs. In this way, model segment 25/17 contained the first $25 \mathrm{nt}$, and the last $17 \mathrm{nt}$ of the S segment anti-genomic strand. All truncations were made using Quikchange mutagenesis (Stratagene), and were confirmed as correct by sequence determination.

\section{Transfections}

Model BUNV segments active for RNA synthesis were generated in BHK cells as previously described (Barr et al. 2003). Briefly, cDNAs encoding the model segment and S and L segment ORFs were transfected into BHK cells previously infected with vaccinia virus recombinant vTF7-3, which expressed T7 RNA polymerase. Resulting BUNV RNAs were harvested using the RNeasy procedure (Qiagen), and used as template for primer extension analysis using ${ }^{33} \mathrm{P}$ end-labeled PAGE-purified oligonucleotides (Invitrogen). Primer extension products were electrophoresed on standard sequencing gels, alongside appropriate sequence ladders to act as size markers (data not shown).

\section{SUPPLEMENTAL MATERIAL}

Supplemental material can be found at http://www.rnajournal.org.

\section{ACKNOWLEDGMENTS}

We thank Dr. Andy Baron of the JIF Centre for Biomechanical Interactions (University of Leeds) for expert assistance with all AUC analysis. The JIF Centre is funded in a major part by a grant from The Wellcome Trust. We also thank Dr. Todd Green (University of Alabama) for assistance with gel filtration analysis, Professor Richard M. Elliott (University of St. Andrews) for continued use of cDNA sequences expressing the BUNV $\mathrm{N}$ protein, and Denise Ashworth (University of Leeds) for sequence analysis. This work was supported by a Research Council UK academic fellowship to J.N.B.

Received September 15, 2008; accepted December 7, 2008.

\section{REFERENCES}

Albertini, A.A., Wernimont, A.K., Muziol, T., Ravelli, R.B., Clapier, C.R., Schoehn, G., Weissenhorn, W., and Ruigrok, R.W.
2006. Crystal structure of the rabies virus nucleoprotein-RNA complex. Science 313: 360-363.

Barr, J.N., Elliott, R.M., Dunn, E.F., and Wertz, G.W. 2003. Segmentspecific terminal sequences of Bunyamwera bunyavirus regulate genome replication. Virology 311: 326-338.

Barr, J.N., Rodgers, J.W., and Wertz, G.W. 2005. The Bunyamwera virus mRNA transcription signal resides within both the $3^{\prime}$ and the $5^{\prime}$ terminal regions and allows ambisense transcription from a model RNA segment. J. Virol. 79: 12602-12607.

Baudin, F., Bach, C., Cusack, S., and Ruigrok, R.W. 1994. Structure of influenza virus RNP. I. Influenza virus nucleoprotein melts secondary structure in panhandle RNA and exposes the bases to the solvent. EMBO J. 13: 3158-3165.

Egelman, E.H., Wu, S.S., Amrein, M., Portner, A., and Murti, G. 1989. The Sendai virus nucleocapsid exists in at least four different helical states. J. Virol. 63: 2233-2243.

Elliott, R.M. 1985. Identification of nonstructural proteins encoded by viruses of the Bunyamwera serogroup (family Bunyaviridae). Virology 143: 119-126.

Elliott, R.M. 1989a. Nucleotide sequence analysis of the large (L) genomic RNA segment of Bunyamwera virus, the prototype of the family Bunyaviridae. Virology 173: 426-436.

Elliott, R.M. 1989b. Nucleotide sequence analysis of the small (S) RNA segment of Bunyamwera virus, the prototype of the family Bunyaviridae. J. Gen. Virol. 70: 1281-1285.

Fuller, F. and Bishop, D.H. 1982. Identification of virus-coded nonstructural polypeptides in bunyavirus-infected cells. J. Virol. 41: 643-648.

Fuller, F., Bhown, A.S., and Bishop, D.H. 1983. Bunyavirus nucleoprotein, N, and a nonstructural protein, NSS, are coded by overlapping reading frames in the S RNA. J. Gen. Virol. 64: 17051714.

Gentsch, J.R. and Bishop, D.L. 1979. M viral RNA segment of bunyaviruses codes for two glycoproteins, G1 and G2. J. Virol. 30: $767-770$.

Green, T.J., Zhang, X., Wertz, G.W., and Luo, M. 2006. Structure of the vesicular stomatitis virus nucleoprotein-RNA complex. Science 313: 357-360.

Gubbay, O., Curran, J., and Kolakofsky, D. 2001. Sendai virus genome synthesis and assembly are coupled: A possible mechanism to promote viral RNA polymerase processivity. J. Gen. Virol. 82: 2895-2903.

Hacker, D., Raju, R., and Kolakofsky, D. 1989. La Crosse virus nucleocapsid protein controls its own synthesis in mosquito cells by encapsidating its mRNA. J. Virol. 63: 5166-5174.

Hacker, D., Rochat, S., and Kolakofsky, D. 1990. Anti-mRNAs in La Crosse bunyavirus-infected cells. J. Virol. 64: 50515057.

Iseni, F., Baudin, F., Blondel, D., and Ruigrok, R.W. 2000. Structure of the RNA inside the vesicular stomatitis virus nucleocapsid. RNA 6: 270-281.

Iseni, F., Baudin, F., Garcin, D., Marq, J.B., Ruigrok, R.W., and Kolakofsky, D. 2002. Chemical modification of nucleotide bases and mRNA editing depend on hexamer or nucleoprotein phase in Sendai virus nucleocapsids. RNA 8: 1056-1067.

Leonard, V.H., Kohl, A., Osborne, J.C., McLees, A., and Elliott, R.M. 2005. Homotypic interaction of Bunyamwera virus nucleocapsid protein. J. Virol. 79: 13166-13172.

Mir, M.A. and Panganiban, A.T. 2004. Trimeric hantavirus nucleocapsid protein binds specifically to the viral RNA panhandle. J. Virol. 78: 8281-8288.

Mir, M.A. and Panganiban, A.T. 2005. The hantavirus nucleocapsid protein recognizes specific features of the viral RNA panhandle and is altered in conformation upon RNA binding. J. Virol. 79: 1824-1835.

Namba, K., Pattanayek, R., and Stubbs, G. 1989. Visualization of protein-nucleic acid interactions in a virus. Refined structure of intact tobacco mosaic virus at $2.9 \AA$ resolution by x-ray fiber diffraction. J. Mol. Biol. 208: 307-325. 
Nugent, C.I., Johnson, K.L., Sarnow, P., and Kirkegaard, K. 1999. Functional coupling between replication and packaging of poliovirus replicon RNA. J. Virol. 73: 427-435.

Ortega, J., Martin-Benito, J., Zurcher, T., Valpuesta, J.M., Carrascosa, J.L., and Ortin, J. 2000. Ultrastructural and functional analyses of recombinant influenza virus ribonucleoproteins suggest dimerization of nucleoprotein during virus amplification. J. Virol. 74: 156-163.

Osborne, J.C. and Elliott, R.M. 2000. RNA binding properties of Bunyamwera virus nucleocapsid protein and selective binding to an element in the $5^{\prime}$ terminus of the negative-sense $S$ segment. J. Virol. 74: 9946-9952.

Rodgers, J.W., Zhou, Q., Green, T.J., Barr, J.N., and Luo, M. 2006. Purification, crystallization and preliminary $\mathrm{x}$-ray crystallographic analysis of the nucleocapsid protein of Bunyamwera virus. Acta Crystallogr. Sect. F Struct. Biol. Cryst. Commun. 62: 361-364.

Schuck, P. 2000. Size-distribution analysis of macromolecules by sedimentation velocity ultracentrifugation and lamm equation modeling. Biophys. J. 78: 1606-1619.
Severson, W.E., Xu, X., and Jonsson, C.B. 2001. cis-Acting signals in encapsidation of Hantaan virus S-segment viral genomic RNA by its N protein. J. Virol. 75: 2646-2652.

Stafford, W.F. and Sherwood, P.J. 2004. Analysis of heterologous interacting systems by sedimentation velocity: Curve fitting algorithms for estimation of sedimentation coefficients, equilibrium and kinetic constants. Biophys. Chem. 108: 231-243.

Valegard, K., Murray, J.B., Stockley, P.G., Stonehouse, N.J., and Liljas, L. 1994. Crystal structure of an RNA bacteriophage coat protein-operator complex. Nature 371: 623-626.

Venter, P.A., Krishna, N.K., and Schneemann, A. 2005. Capsid protein synthesis from replicating RNA directs specific packaging of the genome of a multipartite, positive-strand RNA virus. J. Virol. 79: 6239-6248.

Ye, Q., Krug, R.M., and Tao, Y.J. 2006. The mechanism by which influenza A virus nucleoprotein forms oligomers and binds RNA. Nature 444: 1078-1082.

Zuker, M. 2003. Mfold web server for nucleic acid folding and hybridization prediction. Nucleic Acids Res. 31: 3406-3415. 

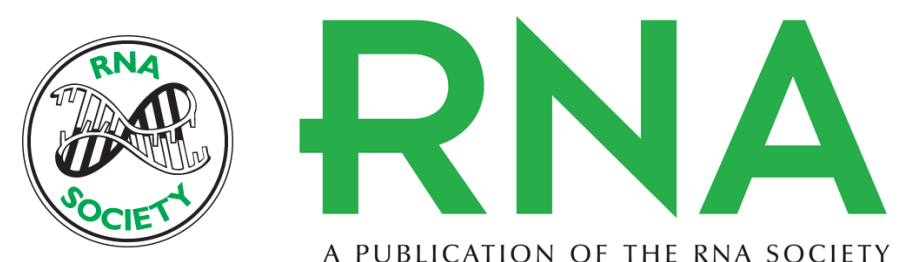

A PUBLICATION OF THE RNA SOCIETY

\section{Investigating the specificity and stoichiometry of RNA binding by the nucleocapsid protein of Bunyamwera virus}

Bjorn-Patrick Mohl and John N. Barr

RNA 2009 15: 391-399 originally published online January 23, 2009

Access the most recent version at doi:10.1261/rna.1367209

\section{Supplemental http://rnajournal.cshlp.org/content/suppl/2009/01/26/rna.1367209.DC1 \\ Material}

References This article cites 32 articles, 18 of which can be accessed free at:

http://rnajournal.cshlp.org/content/15/3/391.full.html\#ref-list-1

\section{License}

Email Alerting Receive free email alerts when new articles cite this article - sign up in the box at the Service top right corner of the article or click here.

\section{IIIII!' Providing Precise Solutions for your research.}

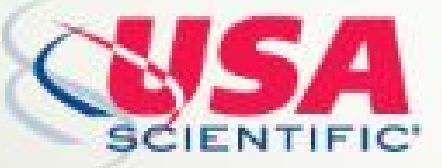

To subscribe to RNA go to:

http://rnajournal.cshlp.org/subscriptions 